\title{
UAV Automatic Test System Design based on VXI-Bus
}

\author{
Dingwen Peng ${ }^{1,2, a}$, Wenling Huang ${ }^{2, b}$ \\ ${ }^{1}$ Dept. of Weapon Engineering, Naval University of Engineering, Wuhan, Hubei, China \\ ${ }^{2}$ No. 4801 Factory, Dongguan, Guangdong, China \\ apengdingwen9@163.com, bhaigonghwl@163.com
}

Keywords: Automatic Test System, CAMAC-bus, VXI-bus

Abstract. In order to meet high precision and strict real-time requirements, an automatic test system design plan is proposed for Unmanned Aerial Vehicle (UAV) with many test items based on VXI-bus. MXI control mode of the VXI-bus system achieves the automated testing of UAV electrical system, control system, guidance system, inertial navigation system and other work status and performances. Actual results show that compared with the original CAMAC-bus system, the test efficiency is improved by $100 \%$. The performance is reliable and work is stable, and it's easy to be extended to the performance testing of other types of UAV.

\section{Introduction}

With the continuously developed technology of UAV and plenty of electronics with new technologies, more and higher requirements are required for UAV test system. Many shortcomings existed in current special test equipment, such as poor generality, vast equipment size, low degree of automation and too many special instruments [1]. Thus, it demands for new test system to fulfill the testing demands of UAV in new models. The automatic test system has experienced the development process from the tailored version to general type. Especially with the occurrence of Bus technology, the automatic test technology entered a new development period, which enables the test system enhancing the degree of automation and integration increasingly [2]. The modules based on the Bus standard enjoys the advantages of low cost and high performance, thus making it widely developed and applied [3].

In comparison with CAMAC-Bus, the VXI-Bus has the advantages such as open system structure, modular design, good electromagnetic compatibility and compact form [4]. By using VXI-Bus it can realize the generalization, modularization and standardization of the test system. The implementation is quite simple with reliable performance [5]. So the VXI-Bus is chosen for the test system. In addition, by utilizing the VXI-Bus interface technology and digital direct frequency synthesis technique, the dual-channel VXI high frequency signal source module is designed.

\section{UAV Working Principle}

\section{Structure Composition and Working Principle.}

The principle diagram for a certain type of UAV is shown in Fig. 1. It consists of:

(1) Power system. Power system is mainly used for generating the force to pull and push the UAV.

(2) Control system. Control system controls the flight attitude and height of the UAV , the working state of guidance system, single electronic equipment of the UAV for self-test. It contains the comprehensive controller, timer and height device etc.

(3) Guidance system. Being the core system of UAV, the guidance system provides the control system with guidance information in the terminal phase in order to improve the accuracy of flight control. It has complex systematical structure, involving the signals of high/low frequency, AC/DC, flight course/ distance etc. It has higher requirement for the reliability, adaptability and expansibility of the automatic test system. The design of guidance system is the most important and difficult part for the design of automatic test system.

(4) Electrical system. Electrical system supplies power source for various electronics inside the UAV, including the cable network connecting various electronics. 
(5) Inertial navigation system. Inertial navigation system provides navigation information for UAV during initial stage and middle section of flight, controls the UAV to fly toward the predetermined area.

(6) Actuator. Actuator includes the power amplifier and steering engine, which receives control message from the control system and converts them into actuator's action, enabling the UAV flying according to requirements.

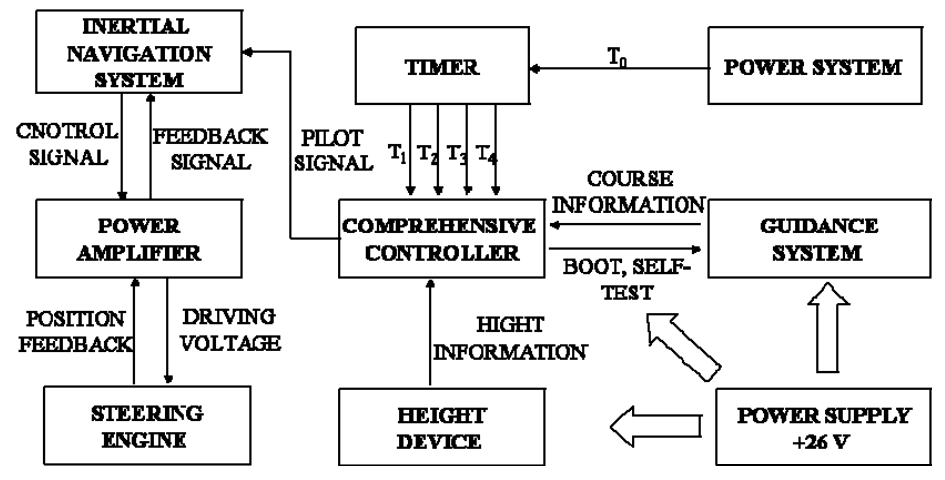

Fig. 1. Principle diagram for certain UAV

\section{Performance Parameter.}

The performance parameters for a certain type of UAV are given below:

(1) DC power supply: $+26 \mathrm{~V} \pm 2 \mathrm{~V}, \pm 40 \mathrm{~V} \pm 3 \mathrm{~V}, \pm 12 \mathrm{~V} \pm 2 \mathrm{~V}$.

(2) Single phase AC power supply: $\sim 32 \mathrm{~V} \pm 3 \mathrm{~V}, \sim 100 \mathrm{~V} \pm 5 \mathrm{~V}$.

(3) Analog output channel (48 ways in total): the main analog including: a) Frequency source operating current $(12 \mathrm{~mA} \sim 20 \mathrm{~mA})$; b) Three ways gyro drift $(0 \sim 0.3 \mathrm{~V})$; c) Course signal $(-10 \mathrm{~V} \sim+10$ $\mathrm{V})$; d) $\mathrm{VCO}$ voltage $(3.0 \mathrm{~V} \sim 8.0 \mathrm{~V})$; e) Height voltage $(0.5 \mathrm{~V} \sim 8.0 \mathrm{~V})$; f) Steering engine feedback voltage $(-5 \mathrm{~V} \sim+5 \mathrm{~V})$; etc.

(4) Switching value output channel (36 ways in total): the main switching value including: a) $1 \# \sim 5 \#$ boot instruction; b) $1 \# \sim 5 \#$ module self-test instruction; c) Frequency source working instruction; d) Six ways control instruction; etc.

(5) Time interval: a) Target delay $(5 \mu \mathrm{s} \pm 0.5 \mu \mathrm{s})$; b) I-command time (20 $\mathrm{s} \pm 0.5 \mathrm{~s})$; c) II-command time ((35 $\mathrm{s} \pm 0.5 \mathrm{~s})$; d) III-command time (480 $\mathrm{s} \pm 5 \mathrm{~s})$; etc.

(6) Dynamic range of frequency source: $500 \mathrm{MHz} 1000 \mathrm{MHz}$.

(7) Flying height: $\mathrm{H}_{0} \mathrm{~m} \sim \mathrm{H}_{1} \mathrm{~m}$.

\section{Bus Design of Automatic Test System}

\section{System Design Requirements.}

According to the principles of standardization, serialization and generalization, the design requirements for the automatic test system are given below:

(1) It creates a general internal/ external work environment for UAV.

(2) It can simulate the working state of every single system in the UAV, ensuring that every system can work well independently, and that the subsystem can be tested respectively.

(3) To meet the requirements of electronic countermeasure test, it may need to design a high frequency source with dual-channel output.

(4) It can real-time monitor main parameters for maintenance personnel to analyze and position faults accordingly.

(5) It shall select the equipments and instruments with expansibility and generality, and easy to be examined and repaired.

The testing requirements shall contain the signals of each system \& parts test for UAV and parameter features, power source and incentive requirements, system volume size and application environment requirement etc. By combining the testing requirements of UAV, the automatic test system shall meet the following test specifications: 
(1) DC power supply: $+26 \mathrm{~V} \pm 1 \mathrm{~V}, \pm 40 \mathrm{~V} \pm 1 \mathrm{~V}, \pm 12 \mathrm{~V} \pm 1 \mathrm{~V}$;

(2) Single phase AC power supply: $\sim 32 \mathrm{~V} \pm 1 \mathrm{~V}, \sim 100 \mathrm{~V} \pm 3 \mathrm{~V}$;

(3) A/D channel: 64 ways $( \pm 1 \%)$;

(4) I/O switching value: 64 ways, input voltage $0 \pm 0.5 \mathrm{~V}$ or $26 \mathrm{~V} \pm 0.5 \mathrm{~V}$;

(5) Time interval: 1 way $( \pm 0.05 \mu \mathrm{s}), 2$ ways $( \pm 0.05 \mathrm{~s}), 1$ way $( \pm 0.2 \mathrm{~s})$;

(6) Signal source frequency: $500 \mathrm{MHz} 1000 \mathrm{MHz}( \pm 0.5 \mathrm{MHz})$;

(7) Signal source power: $+5 \mathrm{~dB} \sim-60 \mathrm{~dB}( \pm 0.4 \mathrm{~dB})$;

(8) Simulated height signal: $\mathrm{H}_{0} \mathrm{~m} \sim \mathrm{H}_{1} \mathrm{~m}(1 \%)$.

\section{System Bus Selection.}

By comparing the CAMAC-Bus with VXI-Bus, it can be seen that:

(1) The data transfer rate of CAMAC Bus is only $3 \mathrm{MB} / \mathrm{s}$, and that of VXI-Bus can be $40 \mathrm{MB} / \mathrm{s}$.

(2) There're totally 4 sizes available for VXI modules and Chassis, and the chassis with bigger size is compatible with the smaller module. However, with only one size for CAMAC Chassis and module, the adaptability for such fields is quite poor with strict requirement for system size.

(3) VXI module shall have faster operation speed, higher measuring accuracy, smaller size and more stable performance than that of CAMAC module.

(4) CAMAC module has no standardized software, and the programmer needs to write the low-level driver program accordingly; VXI module has the corresponding software structure standard, thus the programmer can call the module function through simple program easily.

(5) Because of the restraint factors such as lower transmission rate, CAMAC-Bus may not adapt to the requirements of large-scale automatic test system. In addition, the VXI-Bus system with open structure has the characteristics such as more optional modules, high flexibility and short forming time.

In summary, technical design scheme with the MXI control method that taking VXI-Bus as the system main bus is selected.

\section{System Composition.}

According to the demand analysis of UAV to select hardware equipment, its automatic test system diagram is given in Fig. 2. It mainly contains 6 parts including the computer, VXI device, power supply, special adapter, height simulator, target simulator etc.

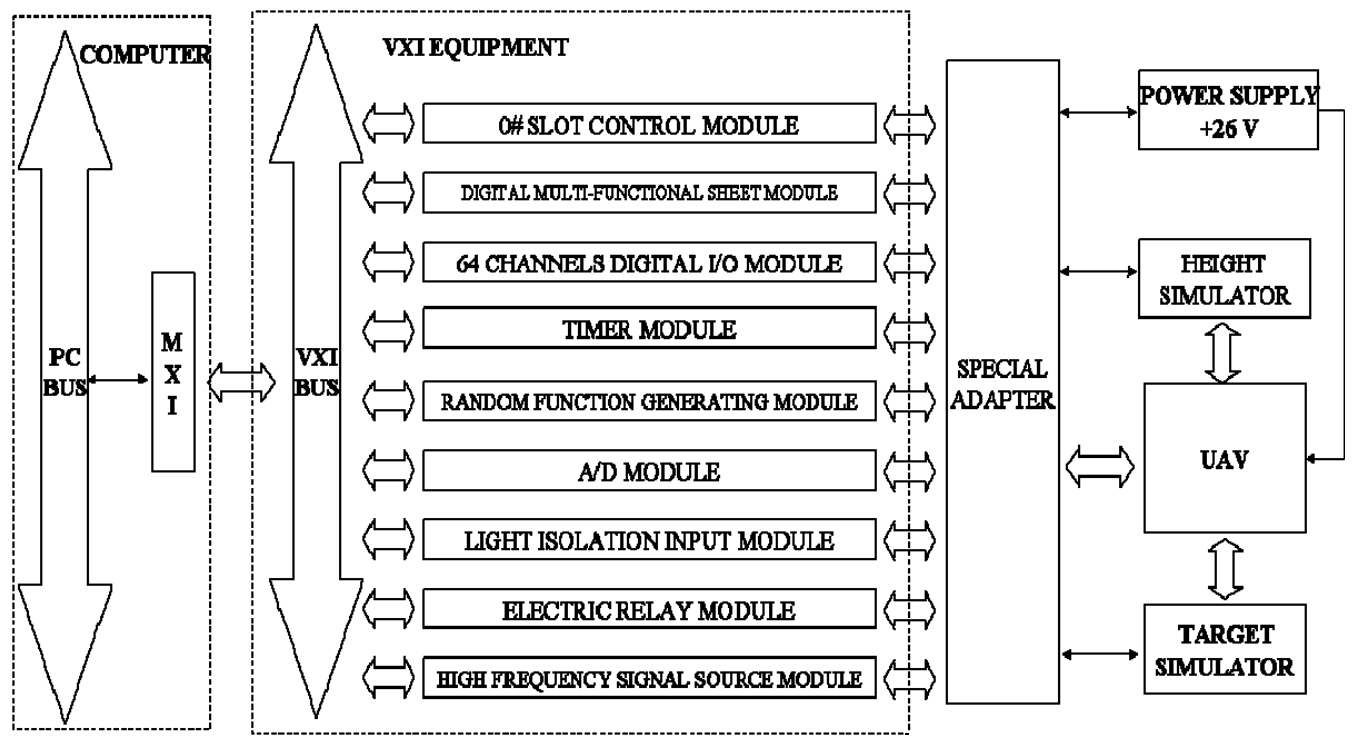

Fig. 2. Automatic test system diagram

The system uses 13 slots inside the VXI chassis and 9 functional modules, including: 0\# slot control module, digital multi-functional sheet module, 64 channel digital I/O module, timer module, random function generating module, $\mathrm{A} / \mathrm{D}$ module, light isolation input module, electric relay module, high frequency signal source module. 
The height simulator is used for simulating the flight height of UAV. The target simulator is used for simulating the target's movement along side the direction.

\section{VXI High Frequency Signal Source Module.}

VXI high frequency signal source module mainly consists of VXI-Bus interface circuit and pulse generating circuit.

(1) Design of VXI-Bus interface circuit. VXI-Bus interface circuit adopts the register based device plan, which mainly performs the transformation between VXI-Bus interface and local bus interface, responses to the read/ write operation of VXI-Bus etc [6]. The composition diagram is shown in Fig. 3. It consists of buffer driving circuit, address bus driving circuit, data bus driving circuit, CPLD. Among them, the CPLD adopts the EPM3256A chip of Altera Company which integrated the functions of configuration register, bus address decoding, address decoding and control logic.

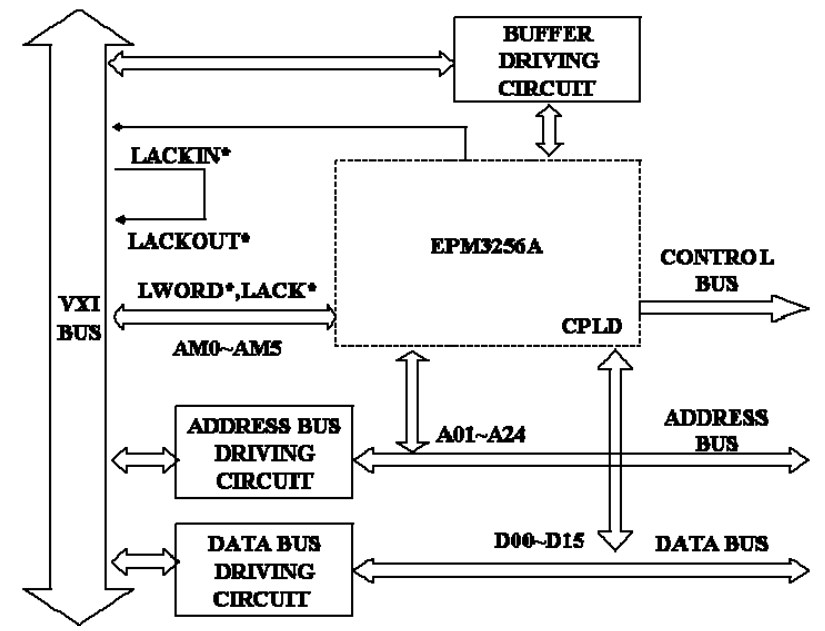

Fig. 3. Register based device

(2) Design of pulse generating circuit. The pulse generating circuit can generate the sinusoidal signal at $500 \mathrm{MHz} \sim 1000 \mathrm{MHz}$, and the adjustable power with modulating signal output. Design of pulse generating circuit mainly includes the following technical proposals: direct frequency synthesizer technique, indirect frequency synthesizer technique and direct digital frequency synthesizer technique (DDS).

DDS has the advantages such as lower power consumption, higher resolution and faster time conversion, so that the system adopted the DDS technical proposal. The DDS device of VXI signal source module used the AD9958 chip of ADI Company, with clock operating frequency $500 \mathrm{MHz}$. It can generate the dual-channel signal with the maximum frequency of $250 \mathrm{MHz}$ [8]. Through frequency multiplier, the maximum output can be $1000 \mathrm{MHz}$.

$$
f_{\mathrm{s}}=\frac{(F T W)^{*} f_{0}}{2^{32}}
$$

Where, $f_{\mathrm{s}}$ is the output frequency; $f_{0}$ is the referenced clock frequency; FTW is the 32-bit frequency control word.

$$
A=\frac{(A C R)^{*} V_{\max }}{2^{10}}
$$

Where, $A$ is the output amplitude; $A C R$ is 10-bit amplitude control word; $V_{\max }$ is the maximum amplitude and can be set with the externally-connected resistance. 


\section{Test Result and Conclusion}

\section{Test Result.}

By testing the above automatic test system, the results showed that both its performance and parameter meet with design requirements, and that it can fulfill the test demands for the UAV. Part of test data are given below:

(1) Maximum error for signal source set frequency: $\leq 0.5 \mathrm{MHz}$;

(2) Maximum error for signal source output: $\leq 0.3 \mathrm{~dB}$;

(3) Error for simulated height: $\leq 0.8 \%$;

(4) A/D channel accuracy: $\leq 0.8 \%$;

(5) Time interval accuracy: 1 way $( \pm 0.02 \mu \mathrm{s}), 2$ ways ( $\pm 0.01 \mathrm{~s}), 1$ way $( \pm 0.1 \mathrm{~s})$;

The main apparatus used in this test are: E4447A spectrum analyzer, KH4137 distortion meter, 34401A digital multimeter, E4418B microwave power meter.

\section{Conclusion}

The following problems are mainly solved:

(1) Through comparison and demonstration between CAMAC-Bus and VXI-Bus, the automatic test system based on VXI-Bus is designed.

(2) The VXI-Bus interface based on CPLD chip is designed, which has realized the docking between signal source and VXI-Bus.

(3) The dual-channel pulse generating circuit based on DDS chip is designed, which solved the restraint problem for the module volume of high frequency source.

With practical application, the stable performance and reliable operation of this system is certified, which can fulfill the test demand of the UAV. All the test results are in accordance with the requirements. At present it has been completed more than 150 items/ times product tests, and the qualified products with such test work well after delivery to the customer with no exception.

\section{References}

[1] Biao Yang, You Gao and Lijuan Yao: Design and Implementation of Automatic Test System Based on VXI-GPIB Bus, Popular Science \& Technology, Vol. 17(2015), p. 14-16 (In Chinese)

[2] Qiang Zhao, SongFeng Liu and Peng Cheng: Development and Key Technology in General Purpose Automatic Test System, Electronic Design Engineering, Vol. 19 (2011), p. 160-162 (In Chinese)

[3] Jianbing Qu: Review of the Development of Military Automatic Test System, Helicopter Technique, Vol. 1 (2014), p. 59-64 (In Chinese)

[4] Junhao Niu, Zhi Li and Chuanpei Xu: Design of Multi-Channel RS485 Module Based on VXI Bus, Computer Measurement \& Control, Vol. 19 (2011), p. 3149-3151 (In Chinese)

[5] Aizhong Sun: Design of VXI Bus Based Automatic Testing System for Two Kinds of Computer Modules, Modern Electronic Technology, Vol. 35 (2012), p. 47-49 (In Chinese)

[6] Lin Cao, Wei Jiang: A Design of VXI Bus Interface Based on Register-based Device, Application Of Electronic Technique, Vol. 37 (2011), p. 103-106 (In Chinese)

[7] Erning Zou, Zhongping Xie: Design and Implementation of the Low Frequency Signal Generator Based on the Method of Direct Digital Frequency Synthesis, Process Automation Instrumentation, Vol. 32 (2011), p. 61-63 (In Chinese) 
[8] Chang Chen: DDS chip AD9958 and its application, Shanxi Electronic Technology, Vol. 6 (2010), p. 16-18 (In Chinese) 